\title{
FORTIFIED PORRIDGE IN MOZAMBIQUE
}

APPLICATION OF THE SUPPLY CHAIN ANALYSIS FOR NUTRITION (SCAN) TOOL

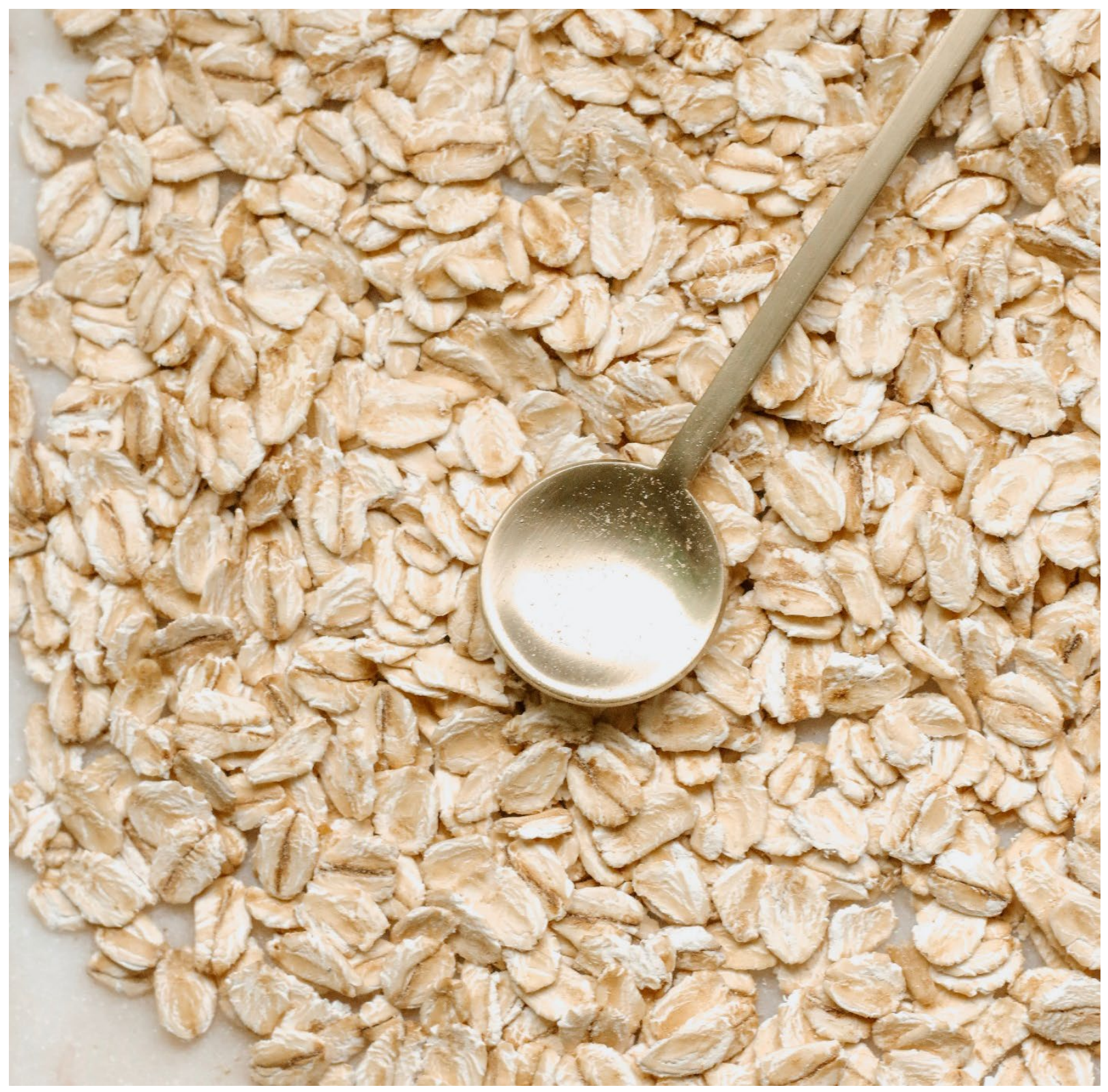

\section{GAIN Briefing Paper $n^{\circ} \mathbf{5}$}

October, 2021

Johanna Farrell

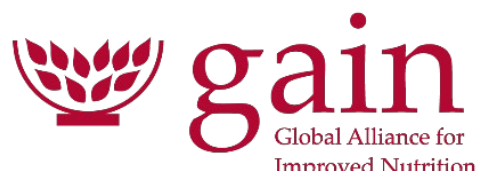




\section{ABOUT GAIN}

The Global Alliance for Improved Nutrition (GAIN) is a Swiss-based foundation launched at the UN in 2002 to tackle the human suffering caused by malnutrition. Working with governments, businesses and civil society, we aim to transform food systems so that they deliver more nutritious food for all people, especially the most vulnerable.

\section{Recommended citation}

Farrell, J. Fortified Porridge in Mozambique. Application of the Supply Chain Analysis for Nutrition (SCAN) Tool. Global Alliance for Improved Nutrition (GAIN). Briefing Paper \#5. Geneva, Switzerland, 2021. DOI:

https://doi.org/10.36072/bp.5

\section{(C) The Global Alliance for Improved Nutrition (GAIN)}

This work is available under the Creative Commons Attribution-Non-Commercial-Share Alike 4.0 IGO licence (CC BY-NC-SA 4.0 IGO; https://creativecommons.org/licenses/by-nc-sa/4.0/). Under the terms of this licence, you may copy, redistribute and adapt the work for non-commercial purposes, provided the work is appropriately cited, as indicated below. In any use of this work, there should be no suggestion that GAIN endorses any specific organisation, products or services. The use of the GAIN logo is not permitted. If you adapt the work, then you must license your work under the same or equivalent Creative Commons license. The contribution of third parties do not necessarily represent the view or opinion of GAIN.

\section{Acknowledgements}

Many thanks to Valerie Friesen, Aimé Kwizera, Stella Nordhagen, and Charlotte Pedersen for the feedback on the drafting of this briefing paper, to ThirdWay Africa whose research for GAIN was used as a basis for the analysis, and to GAIN and Oxford Policy Management who developed the Supply Chain Analysis for Nutrition (SCAN) tool, which was used to structure the analysis. This paper was prepared through the financial support of the Dutch Ministry of Foreign Affairs. All photographs included in this document have been taken with consent for use in publications.

\section{GAIN BRIEFING PAPER SERIES}

GAIN Briefing Papers provide essential information in a succinct, accessible form to support informed decisionmaking by stakeholders in the food system to improve the consumption of nutritious, safe food for all people, especially the most vulnerable.

The Global Alliance for Improved Nutrition (GAIN)

Rue de Varembé 7

1202 Geneva

Switzerland

$\mathrm{T}:$ +41227491850

E: info@gainhealth.org

www.gainhealth.org 


\section{SUMMARY}

Malnutrition among infants and young children is widespread in Mozambique, with high rates of stunting among children under 5 years of age. Micronutrient-fortified porridge can help increase the nutrient content of diets and thus contribute to the fight against malnutrition. To support the strengthening of the sector in Mozambique, this briefing paper presents a supply chain analysis of fortified porridge in Mozambique, with a focus on the barriers to possible mechanisms for scaling.

Results show that at least $54 \%$ of the total population reports buying some form of fortified porridge. Despite limited disposable income, caregivers are buying fortified porridge for their children, even if it means providing a smaller portion daily to reduce the frequency of purchase. All fortified porridge products on the market are imported. The fortified porridge supply chain is complex with distribution channels ranging from traditional to informal, sometimes including illegal trade channels. Developing a local product that would cater to the low-income consumer would require significant longer-term investment with a focus on demand creation and building a larger consumer base, as well as tackling several challenges across the supply chain. In the short term, expanding the volume of imported porridge may be the best solution.

\section{KEY MESSAGES}

- In Mozambique, the total value of the fortified porridge market for older infants and young children in 2017 was USD 6.2 million.

- The key barriers affecting the accessibility, desirability, and quality of fortified porridge at this stage are poor accessibility and affordability in rural areas, the fact that fortified porridge is more 'nice-to-have' than 'must-have' like flours or bread, that all current products on the market fall short of international standards, and that quality is affected by irregular consumption or watered-down portions.

- Key challenges for developing a local fortified porridge product revolve around four key areas 1) sourcing of inputs, 2) producers / millers, 3) distribution, and 4) communication and demand creation.

- An import-based solution may ultimately be the way forward in the short term. This pathway would require further investigation. Alternatively, local production would be feasible if significant investment were made with a long-term perspective, driven by demand creation and social marketing. 


\section{BACKGROUND AND OBJECTIVE}

Malnutrition among infants and young children is widespread in Mozambique, which experiences high rates of stunting and micronutrient deficiencies amongst children under 5 years of age (1-3).

Addressing this through interventions such as food fortification is a priority for the Mozambican government (4). Porridge that has been fortified with vitamins and minerals such as iron, could help fill certain nutrient gaps in the diets of young children and thus help address malnutrition. Currently, fortified porridge in Mozambique is imported; leveraging fortified porridge as an intervention to address malnutrition in Mozambique thus depends on either increasing imports or assessing how the domestic production sector can be developed.

To support the strengthening of this sector, this briefing paper presents a supply chain analysis of fortified porridge in Mozambique, with a focus on the barriers and possible mechanisms for scaling, using the Supply Chain Analysis for Nutrition ( $\underline{\text { SCAN) }}$ tool. The SCAN tool was developed by the Global Alliance for Improved Nutrition (GAIN) for analysing specific supply chain weaknesses or bottlenecks and suggesting potential mechanisms to improve nutrition along the supply chain (6). Using the SCAN tool, this analysis builds upon a value chain analysis of fortified complementary foods with a focus on fortified porridges completed by ThirdWay Africa, a consultancy, for GAIN and based on desk research, stakeholder interviews, and consumer research (5).

\section{FINDINGS}

\section{CHARACTERISTICS OF THE FOOD ENVIRONMENT}

At least $54 \%$ of the total Mozambican population claims they buy some form of fortified porridge (5). In Mozambique, general fortified flours offer basic fortification with vitamins and minerals and are sometimes used to make porridge for younger children; packaged fortified flour mix specifically for porridge (i.e., fortified porridge) is further enriched to offer higher nutritional value. The key characteristics of fortified porridge within the food environment (i.e., accessibility, desirability, and quality) are outcomes of the supply chain, in interaction with the broader food system in Mozambique. These shape consumers' decisions around food acquisition, with implications for their nutrition.

Accessibility: Many fortified porridges are not available in rural or informal markets. While accessibility of fortified porridge amongst low-income consumers is limited, they do have access to fortified flours (5). About $54 \%$ of consumers report buying fortified porridge, particularly Nestlé's Cerelac, for their children aged 6 months to 2 years. ${ }^{1}$ This is the case for every province, including Niassa that is one of the poorest, where up to $70 \%$ of consumers claimed to be buying fortified porridge (5). For consumers, critical factors driving repeat consumption are price, nutritional value, and flavour (in that order of importance). Only higher-income groups focus on flavour and nutritional value above price (5), making price a key influencing factor.

\footnotetext{
${ }^{1}$ The World Health Organization recommends that young children under 6 months of age are exclusively breastfed.
} 
Desirability: Maize flour and wheat-based bread and flours, including fortified ones, are diet staples in Mozambique (5). For example, caregivers prepare porridges for their children based on maize flour (i.e., Top Score, White Star). Enriched fortified porridges are more of an aspirational product that caregivers see as a 'nice-to-have' as opposed to a 'must have' (5). All groups (those who do not buy specific fortified porridges, those who buy fortified flours, and those who buy imported fortified porridge brands) are willing to buy a nutritious and accessible 'easy-to-prepare' porridge (5). Preferred packaging size is $250 \mathrm{~g}$ for fortified porridges (5). Based on a survey, $80 \%$ of low-income consumers find the price of 50-60 MZN to be reasonable for a 250g package of fortified porridge (5). Larger sizes were rejected by some consumers, as low-income groups are used to buying products day-to-day as money is available.

Quality: ThirdWay Africa analysed the nutritional content of the main products on the market, and all the products fell short of meeting the Codex Alimentarius nutritional guidelines for food in some way. Many had violations of the World Health Organization guidelines related to breast-milk substitutes (e.g., contain a claim, showing a picture of a feeding bottle), and all had a problematic recommended serving size (which could displace breastmilk). Despite limited disposable income, mothers and other caregivers of young children are buying fortified porridge even if it means providing a smaller portion daily to reduce the frequency of purchase. This affects quality, as it means that the porridge is consumed irregularly and in watered-down portions, failing to optimally meet a young child's nutritional needs (5).

The key barriers related to the accessibility, desirability, and quality of fortified porridge at this stage are the limited accessibility and affordability in rural areas, the fact that fortified porridge is more 'nice-to-have' than 'must-have', that current products on the market fall short of international standards, and that quality is affected by irregular consumption or watered-down portions.

\section{ASPECTS OF THE SUPPLY CHAIN}

In Mozambique, the total value of the fortified porridge market for older infants and young children in 2017 was MZN 375 million, equivalent to USD 6.2 million (7). This is based solely on the declared imports provided by the Mozambican customs, but the fortified porridge supply chain is complex, with distribution channels ranging from traditional to informal - and sometimes including illegal trade channels (Figure 1) (5). The key characteristics of fortified porridge within the food environment are outcomes of the supply chain, in interaction with the broader food system in Mozambique. These shape consumers' decisions around food acquisition, with implications for their nutrition.

PRODUCTS. Pre-farm and On-farm: Fortified porridges for young children are exclusively imported. Post farm: Fortified porridges on the market include products from brands such as Nestlé (Portugal, South Africa, Middle East), Tiger Brands (South Africa), Hero (Europe), Futurelife (South Africa), and Ninolac (Europe). Most are either Nestlé (Cerelac) or Tiger Brand (Purity) products; although some fortified porridges for children enter the country illegally, the scale of this is assessed to be limited (5). The largest variety of fortified porridges is found in supermarkets and formal markets. Nestlé products such as Cerelac and Nestum dominate the market and are found in the south, centre, and north of Mozambique in both formal and informal markets. The South African Tiger Brand's products, selling under the Purity 
product line, are also sometimes found. All other products are exclusively found in supermarkets in the urban centres in Maputo, Beira, and Nampula.

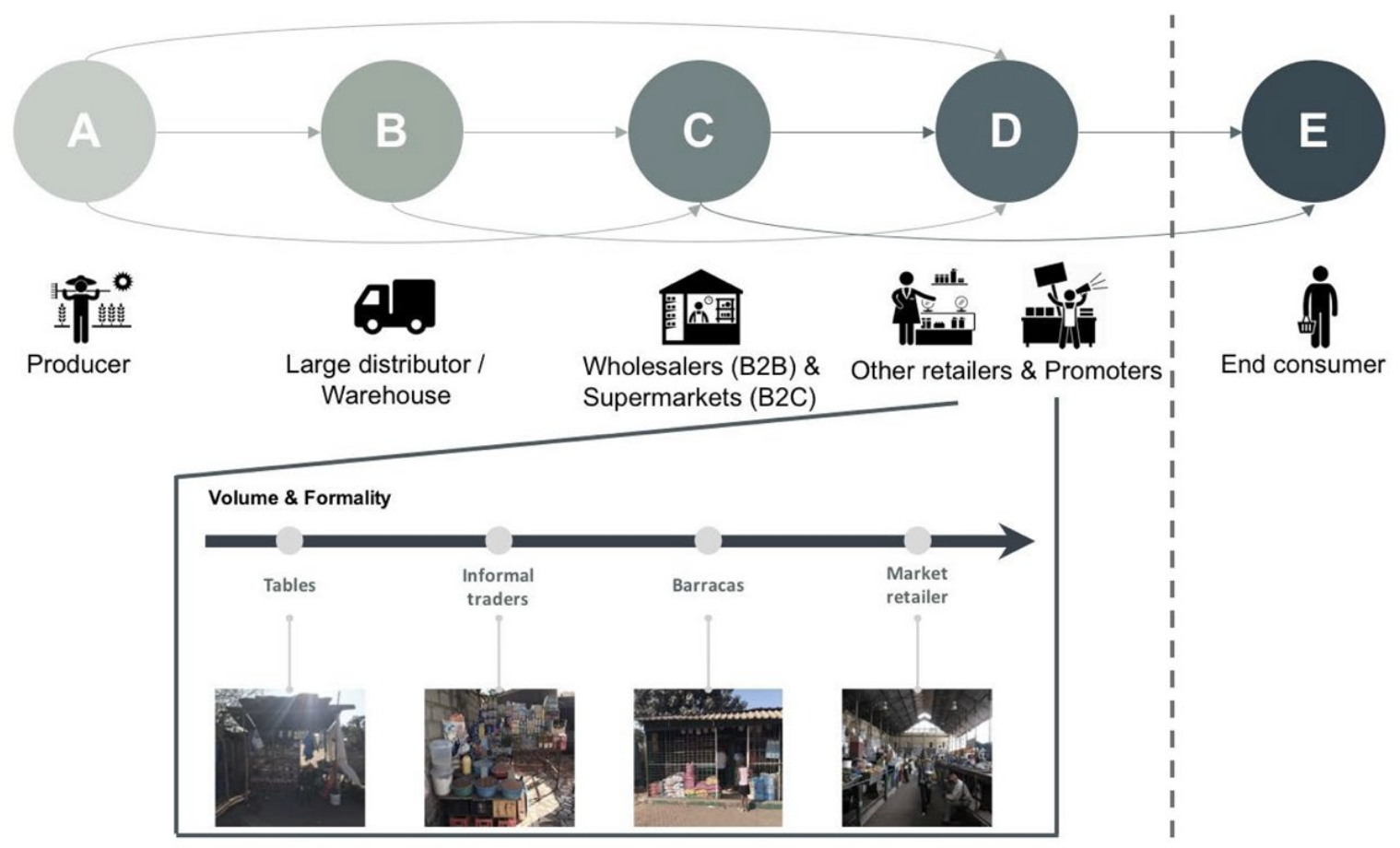

Figure 1: Flow mapping of the fortified porridge value chain in Mozambique (5).

PROCESSES. Pre-farm and On-farm: Increased supply of fortified porridge will result from either increased imports of porridge or from establishing a local industry (likely driven by the private sector). Incentivising local production requires ensuring access to affordable and high-quality raw materials. The most feasible fortified porridge product (based on ThirdWay Africa's analysis) is a maize-and-soyablend type product suitable for older infants and young children. These main macro ingredients are widely available in the country (particularly for maize) and already have a downstream milling industry. Post farm: The establishment of a local industry driven by the private sector, while challenging and requiring patient capital to allow for developing a stable value chain and for demand creation, would be preferable as it could result in positive externalities for the Mozambican economy, such as job creation.

PEOPLE. Pre-farm and On-farm: If a maize-and-soya blend porridge were developed locally, maize and soya farmers would be important players in the supply chain. Smallholders account for almost all of the approximately 2 million tonnes of maize produced in Mozambique; the market for soya is much smaller, and commercial producers account for a larger market, but there are growing numbers of smallholders producing soya (5). Post-harvest losses are an issue for smallholder farmers, who generally use hand-built granaries and other inadequate grain storage structures to store their production. A mid-sized miller could play a key role in addressing this issue by offering centralised storage that would pre-clean the products and store them in bags, at an appropriate moisture level. Post farm: Merec Industries (Maputo and Beira) and CIM (Maputo) are the two larger milling companies in Mozambique and the biggest producers of fortified flour and porridges; they are thus potential producers of local fortified porridge (5). The absence of premix (i.e., the blended vitamin and mineral fortificants to be added to the flour) and packaging producers means future fortified 
producers will be reliant on imports, at least in the short term (5). The lowest income group $(3,500$ MZN - 6,000 MZN (55 - 95 USD) monthly income) is predominant and most reflects the Mozambican demographic reality. While demonstrating price sensitivity, they do buy fortified porridge but may use it irregularly or not in the recommended quantity. Finding a right price point and providing singleserving product offerings will be important to reach this consumer segment (5).

POLICIES. Current fortification legislation does not cover fortified porridge. However, there are a number of relevant laws and guidelines in place for local production of grains, mainly related to product labelling. The current fortified porridge market should follow international standards for fortification (i.e., Codex Alimentarius and WHO), but as noted above, few of the products are currently adhering to these standards, demonstrating a lack of enforcement in the sector (5).

\section{KEY CHALLENGES FOR DEVELOPING THE FORTIFIED PORRIDGE MARKET IN MOZAMBIQUE}

At present, fortified porridge is essentially only imported. Developing a local supply chain could increase availability (and thus help to address malnutrition). Key challenges for developing a local product revolve around four key areas: sourcing of inputs, producers and millers, distribution, and communication and demand creation (Figure 2).

Sourcing of Inputs

- Price-sensitive commodity and availability only 5-6 months a year

- Consistency of raw materials (macro ingredients) sourcing is difficult, due to shortages of maize and soya in certain seasons of the year

- Price volatility is significant

- Local unavailability of some required inputs - Need to import at least pre-mix and quality packaging

\section{Producers / Millers}

- Large producers see the envisaged fortified porridge as a threat to their current brand portfolio

- The cost of a 'ready-to-prepare' maize flour must be competitive with existing maize flour - this will require a very streamlined and lean operation, especially in a market where margins are small

- Lower-income consumers prefer small-sized packages, which reduces the margins due to packaging costs

- Fine-tuning of the porridge flavour requires a great deal of testing prior to launching each new variant

- Lack of technical expertise (with regards to product composition, production, and extrusion processes)

- Small and medium-scale producers are currently using 25 $\mathrm{kg}$ bags. Although smaller-size packaging is preferred by consumers, margins are slim

\section{Distribution}

- Critical mass of potential consumers may be lacking. Rural consumers cannot be considered potential clients due to poor market access, lack of disposable income

- It is easy to import flours/porridges but prohibitive to export

- Market is historically heavily influenced by the informal sector

- Competition from illegal distribution channels: the borders' porosity facilitates illegal entry of competitive substitute products

- Transport infrastructure is poor, increasing transport costs. Security of transport is also an issue as a result of sideselling

- Modern trade channels may not be interested in giving the local porridge shelf space if the product does not turnover quickly. Low volumes will trigger increased margins
Communication and Demand Creation

- Demand creation/market activation will be particularly challenging

- The market has a slow penetration rate, as it is difficult to change consumer habits and preferred brands

- Social marketing campaigns based on formative research are necessary to ensure caregivers understand the added value of the fortified porridge over other products

- Behaviour change interventions are costly and lengthy and require formative research from the start and thorough testing of any planned intervention

- Purchasing power has been diminishing over recent years, and this makes consumers more averse to trying new products

Figure 2: Key challenges for developing fortified porridge market in Mozambique (5). 


\section{RECOMMENDED NEXT STEPS}

In principle, there is willingness to pay for local fortified porridge products. However, whether there is a consumer market with the critical mass that would justify the private sector's required investment is unclear. The large-scale millers need to consider the opportunity cost of diverting part of their production capacity (and a dedicated production line) from mass-volume products towards a product that caters to a niche market. Estimations indicate a total market size of about 80,000 tonnes/year for a porridge destined for older infants and young children. Results show more potential for small- and medium-sized millers to expand their product range into fortified porridges for older infants and young children; with relatively little investment and on a short timeline, they could install production capacity that could cater to a significant market share of consumers. Moreover, local production of fortified porridge is feasible if and only if strong investment is made in demand creation and social marketing from the very beginning, with brand awareness and a reasonable penetration rate achieved in the short- to medium-term.

Sufficient returns for the private sector could potentially be achieved, but a long-term investment perspective is needed (the earliest payback period is 6 or 7 years), as are the financial resources to provide significant cash flow until the breakeven point is achieved. Feasibility could be increased by external support from donors and public-sector stakeholders. Technical support for the formula and consumer testing of the product would be particularly valued by the private sector, in order to get it right from the beginning and reduce costs. Co-investments in social marketing and capital expenditures are other forms of impactful external support. Feasibility would also be increased if production were to start with a porridge marketed to the whole family, not just children, to establish loyalty from a broader customer base.

An import-based solution may ultimately be the way forward in the short term. Supporting such an approach would require further investigation of the pricing of alternative imported fortified porridges as well as their nutritional value and adequacy to address nutritional needs amongst older infants and young children in Mozambique.

\section{CONCLUSIONS}

The key barriers related to the accessibility, desirability, and quality of fortified porridge in Mozambique are the limited accessibility and affordability in rural areas; the fact that fortified porridge is more 'nice-to-have' than 'must-have' like flours or bread; that all current products on the market fall short of international standards; and that quality is affected by irregular consumption or watered-down portions. Additionally, there are several challenges that need to be addressed for a local product to be brought to market, namely in terms of inputs, distribution, millers, and demand creation. Local production would be feasible granted significant investment were made with a longterm investment perspective and driven by demand creation and social marketing. However, an import-based solution may ultimately be the way forward in the short-term. 


\section{REFERENCES}

1. Ministerio da Saúde [MISAU] (2006). National survey on Vitamin A deficiency, anemia and malaria in children 6-59 months and their mothers (December 2001-February 2002). Maputo, Mozambique: MISAU Nutrition Division \& Instituto Nacional de Estadística.

2. MISAU, INE, \& ICFI (2015). Mozambique immunization, malaria, and HIV/AIDS indicators survey 2015. Maputo, Mozambique \& Rockville, MD: National Institute of Health (Mozambique), INE, \& ICFI.

3. MISAU, Instituto Nacional de Estadística [INE], \& ICF International [ICFI] (2013). Mozambique Demographic and Health Survey 2011. Calverton, MD: MISAU, INE, \& ICFI.

4. Amaro, M. Accelerating progress in micronutrient deficiencies in Mozambique: A Ministry of Health perspective. Matern Child Nutr. 2019; 15( S1):e12707. https://doi.org/10.1111/mcn.12707

5. ThirdWay Africa, Fortified Complementary Food - Deliverable 4: Final Report. 2018.

6. The Global Alliance for Improved Nutrition (GAIN), et al: Supply Chain Analysis for Nutrition tool (SCAN). 2020. https://www.gainhealth.org/resources/reports-and-publications/supply-chainanalysis-nutrition-tool-scan.

7. Mozambique Customs Authority, Fortified porridge imports recorded, 2017. 